\title{
New Wine into New Wineskins: Radial Endobronchial Ultrasound for Parenchymal Lung Infiltration
}

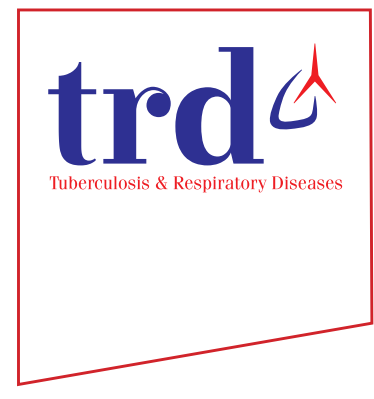

\author{
Kyungjong Lee, M.D. ${ }^{(1)}$ \\ Division of Pulmonary and Critical Care Medicine, Department of Medicine, Samsung Medical Center, Sungkyunkwan \\ University School of Medicine, Seoul, Korea
}

\begin{abstract}
Diagnosis of patients with diffuse lung infiltration is usually difficult because it is not easy to collect lung tissue. A variety of disease manifestations should be considered in patients who have infiltrative parenchymal lung disease, including diffuse lung infection caused by Mycobacterium or fungus, interstitial lung disease, an infiltrative type of malignancy, or noninfectious parenchymal inflammation. To differentiate and diagnose these phenotypes using chest computed tomography, clinicians must decide whether to perform a bronchoscopic lung biopsy in addition to history taking and pulmonary sputum and blood studies.

A bronchoscopic lung biopsy, usually called a transbronchial lung biopsy (TBLB), is a universal technique to obtain tissue from the lung parenchyma by flexible bronchoscopy ${ }^{1}$. Although this technique is safer than a lung resection by video-assisted thoracic surgery, several complications have been reported to accompany this technique, such as bleeding and pneumothorax. The incidence of mild to severe bleeding after lung biopsy is about $1 \%-9 \%$ of patients ${ }^{2,3}$; severe bleeding may interrupt the procedure and result in no tissue for the diagnosis. The incidence of pneumothorax has been reported to be between 1 and $4 \%$ after lung biopsy ${ }^{4-6}$.

TBLB under fluoroscopic guidance has been used to local-
\end{abstract}

Address for correspondence: Kyungjong Lee, M.D.

Division of Pulmonary and Critical Care Medicine, Department of

Medicine, Samsung Medical Center, Sungkyunkwan University School of

Medicine, 81 Irwon-ro, Gangnam-gu, Seoul 06351, Korea

Phone: 82-2-3410-0777, Fax: 82-2-3410-3849

E-mail: kj2011.lee@samsung.com

Received: May. 30, 2019

Revised: Jun. 10, 2019

Accepted: Jun. 18, 2019

(9) It is identical to the Creative Commons Attribution Non-Commercial License (http://creativecommons.org/licenses/by-nc/4.0/). ize target lesions found on chest X-rays. Fluoroscopic guidance of the procedure can diminish the development of postbiopsy pneumothorax. Although TBLB can be performed without fluoroscopy, it is reasonable to use fluoroscopy to obtain lung tissue in cases of localized parenchymal lung diseases involving one or two segments of the lung. Despite these advantages, many bronchoscopy suites do not have fluoroscopic equipment due to the additional cost and having limited space. Another reason for not adopting fluoroscopy is concern about radiation exposure.

In a retrospective study ${ }^{7}$ issued by Tuberculosis and Respiratory Diseases, the authors reported the diagnostic yields of radial probe-endobronchial ultrasound (RP-EBUS)-guided lung biopsy compared to blinded lung biopsy without fluoroscopic assistance. RP-EBUS has been developed to detect peripheral lung nodules and obtain tissue from them ${ }^{8}$. This equipment enables the bronchoscopist to detect an abnormal lung lesion with surrounding vessels and the bronchus. In addition, it can reach localized parenchymal lung, involving one segment, to obtain tissue with biopsy forceps after confirming the diseased parenchyma of the lung through a radial probe. Although it might be anticipated that RP-EBUS-guided lung biopsy would be better for obtaining lung tissue, the study found that the diagnostic yields of blinded and RP-EBUSguided lung biopsy were similar. This can be explained by the observation that diffuse lung infiltration is caused mainly by parenchymal inflammation, which the authors defined as non-diagnostic if the pathologist reported chronic inflammation or interstitial inflammation. Organizing pneumonia, granulomatous inflammation with the detection of acid-fast bacilli or tuberculous polymerase chain reaction and malignancy were considered diagnostic. The TBLB accounted for almost $41.8 \%$ and $41.7 \%$ of chronic inflammation in the RPEBUS TBLB and blind TBLB groups, respectively. However, the diagnostic yield of organizing pneumonia was higher in the RP-EBUS TBLB group (31.3\%) than in the blind TBLB group (18.3\%). Granulomatous inflammation or malignancy, etc. comprised $33 \%$ in the prevalence of pathology. While 
the diagnostic yield of RP-EBUS-guided lung biopsy was not superior to that of blinded lung biopsy, the incidence of pneumothorax may be lower using RP-EBUS-guided lung biopsy. RP-EBUS-guided lung biopsy has several advantages for diagnosing lung parenchymal disease. The bronchoscopist does not have to worry about the radiation hazard associated with fluoroscopic guidance, and RP-EBUS-guided lung biopsy can be performed in a limited space because it does not involve the large amount of equipment needed for fluoroscopy. This retrospective study included only patients with more than one involved lung segment. However, if the involved lung has subsegmental or sub-sub segmental infiltration, the diagnostic accuracy of RP-EBUS-guided lung biopsy might surpass the yield of blinded lung biopsy without fluoroscopic assistance. Although the study did not show the superiority of RP-EBUS guidance for diagnosing infiltrative lung disease localized in more than one lung segment, it implies that clinical implementation of this RB-EBUS technique would be very useful and safe in patients who have localized lung infiltrates.

\section{Conflicts of Interest}

No potential conflict of interest relevant to this article was reported.

\section{References}

1. Hanson RR, Zavala DC, Rhodes ML, Keim LW, Smith JD. Transbronchial biopsy via flexible fiberoptic bronchoscope: results in 164 patients. Am Rev Respir Dis 1976;114:67-72.

2. Zavala DC. Pulmonary hemorrhage in fiberoptic transbronchial biopsy. Chest 1976;70:584-8.

3. Herf SM, Suratt PM, Arora NS. Deaths and complications associated with transbronchial lung biopsy. Am Rev Respir Dis 1977;115:708-11.

4. de Fenoyl O, Capron F, Lebeau B, Rochemaure J. Transbronchial biopsy without fluoroscopy: a five year experience in outpatients. Thorax 1989;44:956-9.

5. Frazier WD, Pope TL Jr, Findley LJ. Pneumothorax following transbronchial biopsy: low diagnostic yield with routine chest roentgenograms. Chest 1990;97:539-40.

6. Hernandez Blasco L, Sanchez Hernandez IM, Villena Garrido V, de Miguel Poch E, Nunez Delgado M, Alfaro Abreu J. Safety of the transbronchial biopsy in outpatients. Chest 1991;99:562-5.

7. Kim EJ, Kim KC. Utility of radial probe endobronchial ultrasound-guided transbronchial lung biopsy in diffuse lung lesions. Tuberc Respir Dis 2019;82:201-10.

8. Mudambi L, Ost DE. Advanced bronchoscopic techniques for the diagnosis of peripheral pulmonary lesions. Curr Opin Pulm Med 2016;22:309-18. 